\title{
El cultivo de carambolo (Averrhoa carambola L.) y su comportamiento en el piedemonte del Meta (Colombia). Una revisión
}

\section{Growing starfruit (Averrhoa carambola L.) and its behavior in the piedmont of Meta (Colombia). A review}
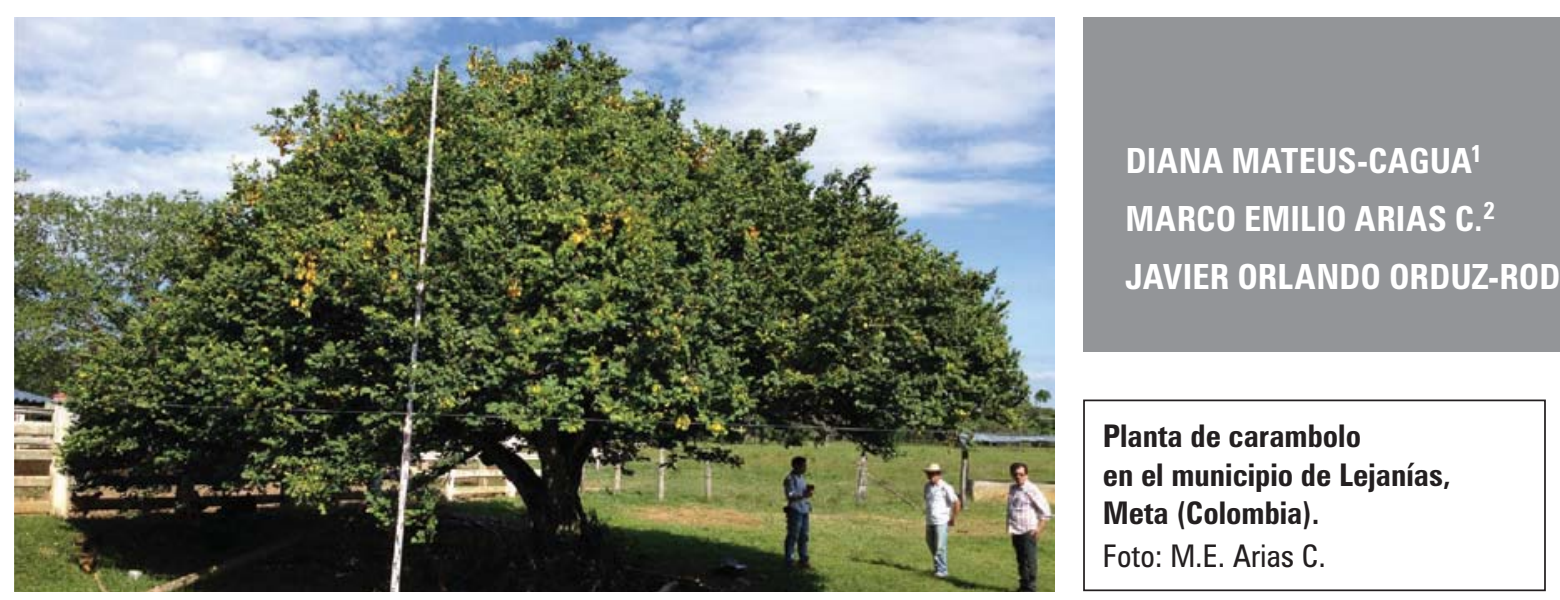

\section{RESUMEN}

Uno de los productos de autoconsumo de los agricultores en el departamento del Meta es el carambolo, llamado también por algunas personas "tamarindo". Es un fruto tropical exótico que ha sido establecido en fincas para consumo en fresco y jugos pero no ha logrado posicionar un nicho de mercado debido al desconocimiento técnico de su manejo y de las ventajas nutricionales que posee. Es popular por su forma de estrella y recomendado para personas con diabetes e hipertensión arterial; para conservar la visión y el buen funcionamiento del sistema inmunológico. La revisión permitió encontrar que este fruto tiene una alta actividad antioxidante, bajo valor calórico, buen contenido de vitamina A y C y minerales como calcio, fósforo y potasio de importancia en la nutrición humana. Sin embargo, debido al contenido de potasio y oxalato de calcio se recomienda un consumo moderado en personas con problemas renales. Las bondades nutricionales y las experiencias de otros países en la poscosecha de estos frutos proveen información importante para ampliar las posibilidades de comercialización del carambolo en condiciones del piedemonte del Meta. Se presenta una breve descripción en las principales localidades donde se han establecido el cultivo, su uso y las formas de comercialización. La información señala que hay posibilidades de mercadeo aunque se debe innovar en el uso que se promueve de tal forma que además del consumo familiar pueda servir para generar excedentes económicos, mejorando las prácticas de manejo y fomentando nichos de mercado (posicionamiento en mercados especializados).

\footnotetext{
Centro de Investigación La Libertad, Corporación Colombiana de Investigación Agropecuaria (Corpoica), Villavicencio (Colombia).

2 Facultad de Ciencias Agropecuarias y Recursos Naturales, Universidad de los Llanos, Villavicencio (Colombia).

3 Autor para correspondencia. jorduz@corpoica.org.co
} 


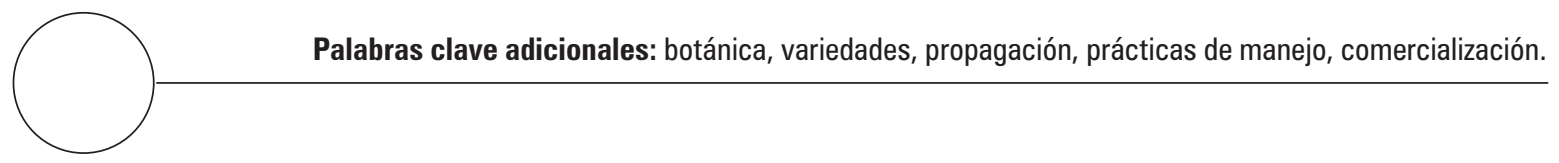

\section{ABSTRACT}

One of the more known subsistence products in the department of Meta is the starfruit, misnamed by some people as tamarind. It is an exotic tropical fruit that has been established on farms for fresh consumption and juices, but it has failed to position itself in a market niche due to the lack of technical management and nutritional advantages. It is popular due to its star shape and recommended for people with diabetes and hypertension to maintain vision and aid proper functioning of the immune system. This review led to the discovery that this fruit has a high antioxidant activity, low calorific value, and good contents of vitamins $A$ and $C$ and minerals such as calcium, phosphorus and potassium, characteristics that are important to health. However, due to the potassium and calcium oxalate contents, moderate or zero consumption is recommended for people with kidney problems. The nutritional benefits and experiences of other countries in the post-harvest of these fruits have provided accurate information on the marketability of starfruits under the conditions of the Meta piedmont. This review presents a brief description of the principal zones where this crop has been established. The information indicates that there is scope for marketing, but innovation in this fruit's use should be promoted so that it, in addition to household consumption, can serve as financial support for farmers. This requires the use of technical management practices and the promotion of market niches.

Additional key words: botanic, varieties, propagation, management practices, commercialization.

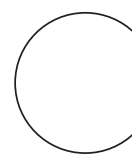

El carambolo (Averrhoa carambola L.), es una fruta de origen tropical originario del suroeste asiático (Janick y Paull, 2008) de la familia Oxalidaceae que puede encontrarse también en condiciones del subtrópico (González et al., 2001). Se conoce como: carambolo (Colombia), lima de cayena (Brasil), árbol de pepino (México), yangt o duraznero extranjero (China), starfruit (Estados Unidos), kamrakh (India), babingbing (Filipinas), tamarindo chino, tiriguro, entre otros (Janick y Paull, 2008; Hii y Ogugo, 2014).

Fue introducido al continente americano a fines del siglo XVIII, y traído a Colombia cerca del año
1930 a la granja experimental de Palmira (Enciclopedia Agropecuaria Terranova, 1995). Se cultiva en diversas regiones tropicales y subtropicales cálidas, en países como: China, Indochina, Malasia, Brasil, México, Tailandia, Estados Unidos, Filipinas, Hawai, Haití y Colombia, aunque no en todos se hace de forma comercial (Nakasone y Paull, 1998; Illescas et al., 2007). Esta planta se encuentra distribuida en una amplia gama de departamentos de Colombia (Valle del Cauca, Córdoba, Antioquia, Quindío, Tolima, Meta, zona Caribe, etc.), aunque no en grandes extensiones (Villegas, 1998). España, Canadá, Francia, Alemania y Japón son los principales mercados (Novillo, 2009). 
Esta especie se encuentra establecida en fincas de pequeños productores en la región del piedemonte del Meta (Colombia) para consumo familiar $y$ venta en pequeños supermercados, aunque algunas veces su distribución es limitada por daños externos a causa de la manipulación de los frutos. Con el propósito de identificar sus potencialidades productivas y de mercadeo, sumado a los diversos beneficios para la salud humana, se desarrolló la presente revisión y un recorrido por la principal zona frutícola del departamento para conocer el estado actual de los cultivos. Se incluyeron temas de ecología del cultivo el cual permitirá mejorar las prácticas agrícolas para incrementar la producción junto con el beneficio económico de los agricultores de la región, e innovación en la transformación del fruto en otros países para abrir mercados especializados tomando como ventaja su apariencia y contenido

\section{ECOLOGÍA DEL CULTIVO}

El carambolo se desarrolla de forma óptima en condiciones del trópico bajo y medio (0-1.200 $\mathrm{msnm}$ ), donde las lluvias sean medias a altas bien distribuidas, alrededor de 1.800-3.000 mm año-1 , pues la falta prolongada de agua limita el crecimiento de las raíces, hojas, desarrollo en general, la inducción de floración y puede reducir significativamente el rendimiento (Paull y Duarte, 2012; Galán y Menini, 1993). Aunque también se han establecido en trópico seco y regiones subtropicales con buenos resultados con excepción de zonas donde se presenten heladas severas (Campbell et al., 1985; Campbell y Malo, 1981). Tiene un óptimo desarrollo en climas húmedos aunque se reporta tolerancia en zonas secas con sequías estacionales (Pérez et al., 2005). La temperatura óptima para este cultivo se encuentra en un rango de 21 a $32^{\circ} \mathrm{C}$ (Wahab et al., 1989). Árboles expuestos a temperaturas de $-2^{\circ} \mathrm{C}$ o menos por largos periodos pueden verse seriamente afectados, desde una completa defoliación hasta la muerte (Sharma et al., 2009; Galán y Menini, 1993). Zonas con alta radiación solar, superior a 2.000 horas de brillo solar/año son recomendadas pues promueven altas concentraciones de solidos solubles en los frutos (George et al., 1988).

Se puede adaptar a suelos desde moderadamente ácidos a suelos neutrales (pH 4,5 a 7,0) (Green, 1987; Crane, 1993); de acuerdo con Al-Yahyai (2004) los árboles de carambolo crecen vigorosamente en suelos ácidos de climas tropicales. Se desarrolla de forma óptima en suelos con textura franca, o ligeramente areno arcillosa, con limitaciones en suelos con problemas de encharcamiento y alta salinidad (Campbell et al., 1985; MACIA, 2003).

\section{DESCRIPCIÓN BOTÁNICA}

Es un arbusto perennifolio (figura 1) que puede alcanzar hasta $10 \mathrm{~m}$ de altura (Saghir et al., 2013). En su estado juvenil tiene porte piramidal mientras que en su estado adulto presenta una copa abierta-irregular; comúnmente muy ramificado, aunque puede tener un tronco simple (Litz y Griffins, 1989; Orduz y Rangel, 2002; Campbell et al.,, 1985); el arbusto es siempre verde y su crecimiento es más lento donde la velocidad del viento es mayor, aunque puede resistir los vientos fuertes ya que sus raíces tienen un anclaje profundo.

El tallo es generalmente torcido, con ramas bajas y delgadas; su diámetro puede alcanzar los 20-30 cm, con corteza de color gris pardo a verde (Villegas, 1998). Las hojas son alternas, compuestas, imparipinnadas, con 5-11 hojuelas alternas o parcialmente opuestas ovadas o elípticas de color verde a verde claro. Los foliolos miden entre 2 y $9 \mathrm{~cm}$ de largo, y 1 y $5 \mathrm{~cm}$ de ancho. Son sensibles a la luz plegándose en las horas de la noche (Orwa et al., 2009).

Las flores son de tonalidades rojas y purpuras agrupadas en inflorescencias racimosas (figura 2A), de tamaño pequeño, perfectas, de pedicelo corto, regulares y con heterostilia o distilia 


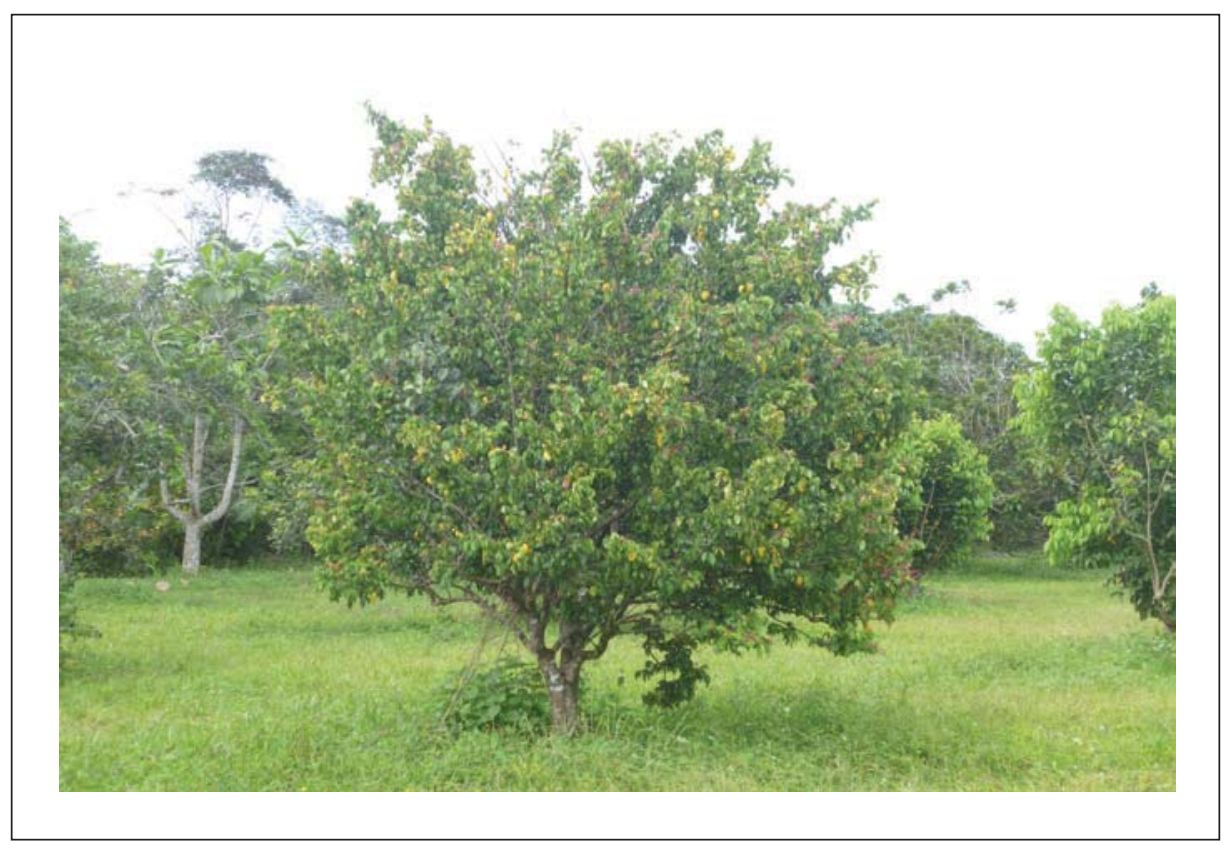

Figura 1. Planta de carambolo en lote de frutales exóticos del Centro de Investigación La Libertad, Corpoica, Villavicencio (Meta). Foto: H. Velásquez.

(algunos árboles producen flores son con estilos cortos y estambres largos, y otros producen flores con estilos largos y estambres cortos; típico de la especie) hermafroditas, autoincompatibles y de polinización cruzada, conformadas por 5 pétalos, 5 sépalos, 5 estambres, 5 estaminodios (Ray, 2002; Knight, 1965; Mendoza y Ramírez, 1997). Abren gradualmente en la mañana entre 8 y 10 a.m. y cierran en horas de la tarde entre 2 y 6 p.m., durante este tiempo los agentes polinizadores (Apis mellifera y Trigona sp.) realizan su labor. La presencia de lluvias fuertes durante el periodo de floración puede afectar de forma negativa la polinización (Ray, 2002; Morton, 1987). Para aumentar el porcentaje de polinización Ngah et al. (1989) recomiendan establecer una colmena por cada 0,4 ha de carambola. También se ha recomendado sembrar más de un cultivar para asegurar la polinización cruzada. Según González (2000), menos del 25\% de las panículas presentan frutos cuajados y regularmente se desarrolla solo un fruto por panícula, este valor es suficiente para obtener una cosecha adecuada.
Orduz y Rangel (2002) afirman que entre siembra y germinación se cuentan 34 d; de germinación a vivero 39 d; de establecimiento en sitio definitivo a floración, 8 a 22 meses después de trasplante; de floración a aparición de primeros frutos 30 días; de aparición de primeras flores a cosecha 120 d. Mientras que Crane (1994) reporta 60-75 d de la floración a maduración de frutos en Florida, tiempo sujeto a la variedad y prácticas de manejo. González et al. (2001) afirman que el fruto presenta un patrón de crecimiento sigmoidal simple con tres fases, y que durante este periodo de desarrollo el tamaño, peso, porcentaje de pulpa, relación de SST y la ATT aumentan mientras que el porcentaje de corteza, la firmeza y acidez del fruto disminuyen. El tiempo estimado entre fruto cuajado y maduro es de 83 d en Perú (Enciclopedia Agropecuaria Terranova, 1995).

El fruto es una baya carnosa dorada en su etapa madura con una cutícula cerosa de color translúcido o dorado pálido que puede medir entre 
5-15 cm de longitud y 3-6 $\mathrm{cm}$ de ancho (figura 2B; Patil et al., 2012). La forma de estrella es característica de este fruto debido a los 4-5 vértices $\mathrm{o}$ aristas longitudinales y redondeadas que siempre están presentes (Ruehle, 1958; Litz y Griffis, 1989). A cada costilla del fruto corresponde un lóculo con dos semillas planas (las cuales pierden viabilidad rápidamente una vez se han extraído del fruto), en total puede contener hasta 12 semillas, aunque se presentan casos de frutos partenocárpicos (León, 2000; Loor, 2013). La pulpa tiene color amarillo claro a oscuro, es crujiente y jugosa; el sabor varía desde muy ácido con poco azúcar a muy dulce con poca acidez, esto depende en medida de la variedad utilizada, copa-patrón y las condiciones ambientales (León, 2000; Nakasone y Paull, 1998; George y Nissen, 1994).

De acuerdo con Galán (1993), el peso recomendado para comercialización en fresco se encuentra entre 100 y $300 \mathrm{~g}$. Se estima que alrededor del $95 \%$ del fruto es comestible, siendo en promedio el $70 \%$ pulpa, el $25 \%$ corteza y $5 \%$ semilla. E1 contenido de agua varía entre $60 \%$ y $80 \%$, los sólidos varían desde 5-14\% y los azúcares totales entre 4 y 11; la concentración de azúcares permanece relativamente constante una vez cosechados los frutos (O'hare, 1997; Campbell y Koch, 1989; Wagner et al., 1975).

\section{VARIEDADES}

A nivel mundial existe un amplio número de variedades, aunque muchas de ellas no son comerciales debido a sus características de calidad. Se acepta de forma general que existen dos tipos principales de fruto de carambolo: dulces y acidas. Las primeras de mayor tamaño, de consistencia blanda y con menos contenido de ácido oxálico (Crane, 1994; Navarro, 2011).

Algunas variedades conocidas son: Arkin (comercialmente importante en Florida), Golden Star, Kaput, Newcombe, Kaput, Cheng-Tsey, B-2, B-10, B-17, entre otras; se estima que el departamento de agricultura de Malasia ha seleccionado más de 17 variedades de interés comercial (MACIA, 2003). El Instituto Colombiano Agropecuario (ICA) seleccionó la variedad Icambola en el año 1972 para Colombia, la cual se adapta a condiciones climáticas del trópico en general. Se busca con el desarrollo de estas varie-

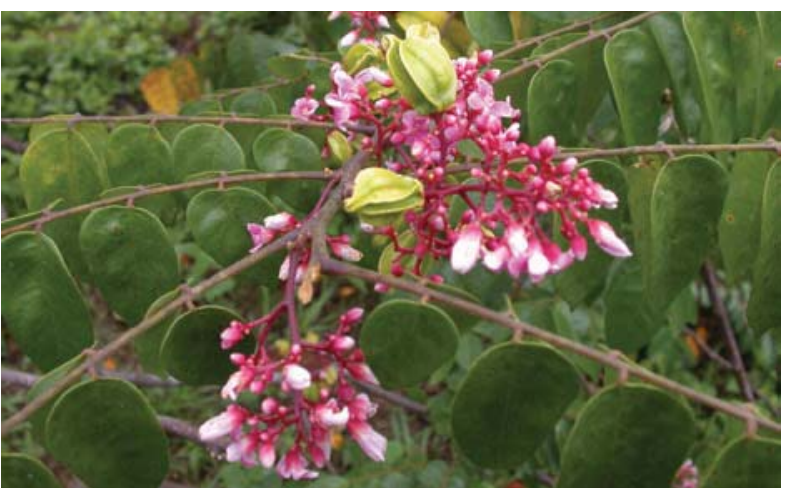

$2 A$

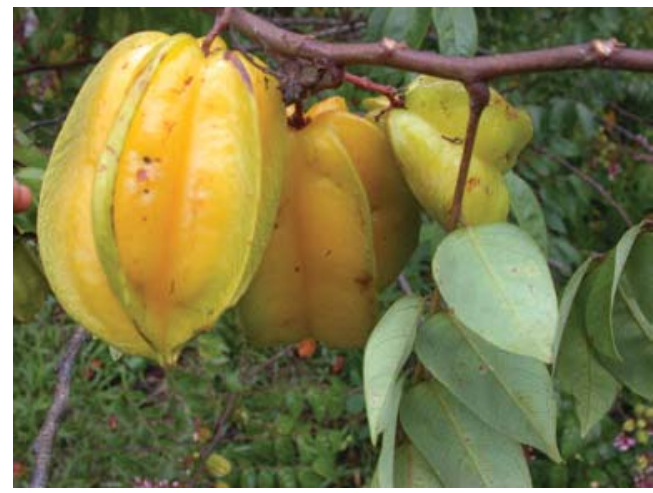

2B

Figura 2. A. Flores de carambolo en racimo, B. Frutos en proceso de maduración en lote de frutales exóticos del Centro de Investigación La Libertad, Corpoica, Villavicencio (Meta). Fotos: H. Velásquez. 
dades frutos con color atractivo (amarillo dorado), vida comercial mayor a tres semanas luego de cosechado, y altas producciones.

\section{SISTEMAS DE PROPAGACIÓN}

Estas plantas pueden ser propagadas sexual o asexualmente (injertos de chapa, púa lateral, de bisel o doble bisel).

\section{Propagación sexual}

Se realiza por medio de semillas, las cuales deben ser seleccionadas de árboles con buena producción y calidad de fruto una vez se han desarrollado por completo. Las semillas deben ser sembradas rápidamente para evitar pérdida de viabilidad, previa separación del arilo que las recubre secándolas en un ambiente bien ventilado y sin luz directa. De acuerdo con Galán y Menini (1993) si las semillas se conservan en agua destilada, en la oscuridad, en un rango de temperatura entre los $28^{\circ} \mathrm{C}$ y los $30^{\circ} \mathrm{C}$ la viabilidad de las mismas puede ser hasta de 24 días; mientras que Paull y Duarte (2012) afirman que las semillas pueden ser almacenadas durante dos semanas si se conservan en refrigeración, estas germinan entre los 8-18 días de sembradas dependiendo del comportamiento ambiental (humedad principalmente). Una de las desventajas de este método es la heterogeneidad que puede resultar en la población además de la entrada más tardía a producción. El material proveniente de este método tiene como destino la producción de portainjertos.

\section{Propagación asexual}

Este se utiliza básicamente en explotaciones comerciales. Los dos tipos de injerto más recomendados son el lateral y el de chapa, en condiciones favorables de temperatura, los brotes se desarrollan entre la semana 3-4 y las copas de los portainjertos pueden cubrirse entre la semana 2 y 3 después de insertar la yema (Galán y Menini, 1993; NIIR Board, 2005; Paull y Duarte, 2012).
También es posible multiplicar por medio de acodos, aunque este último ha mostrado una lenta formación de raíces (Morton, 1987). El trasplante debe realizarse cuando la primera hoja verdadera ha madurado.

\section{SISTEMA DE CULTIVO}

Una vez estudiada la oferta ambiental de la zona y las exigencias propias de esta especie, se toma la decisión de establecer o no el cultivo. Hay diversas recomendaciones de distancia de siembra $(4 \times 6,5 \times 5,6 \times 6,5 \times 7,8 \times 8 \mathrm{~m}$, etc.), en sistemas de tresbolillo, cuadro o rectángulo, el primero recomendado para laderas y los dos últimos preferiblemente para terrenos planos o ligeramente inclinados. Las distancias dependerán del objetivo de la plantación, Campbell (1985) menciona que estos pueden ser intercalados con otros cultivos arbóreos o herbáceos como la papaya. Comercialmente se manejan densidades entre 290 y 360 plantas/ha (Galán y Menini, 1993; Crane, 1994).

De acuerdo con Campbell (1985), el sombreado de la copa del árbol reducirá la producción de fruta, por lo cual las filas deben orientarse de tal forma que las plantas reciban la mayor exposición a la luz solar (Campbell y Marte, 1990). En condiciones del trópico las plantas se desarrollan de forma vigorosa requiriendo podas anuales para el control del tamaño y uso eficiente de la radiación, estas podas por lo general estimulan la floración (Núñez-Elisea y Crane, 2000). Se recomienda hacer poda en la parte inferior del árbol para evitar ramas a menos de un metro de cercanía al suelo para evitar daños en los frutos (Galán y Menini, 1993), también son necesarias en plantaciones comerciales cuando los árboles superan los 4 metros.

\section{COSECHA Y POSCOSECHA}

Los frutos se recolectan manualmente preferiblemente maduros, ya que en poscosecha estos tienen un proceso mucho más lento para alcan- 
zar color aunque sigue perdiendo clorofila y desarrollando carotenoides, mientras que el contenido de azucares no varía luego de recolectado, es no climatérico (O'Hare, 1993; Bouzayen et al., 2010). El punto de recolección comercial es el cambio de color de verde a verde amarillento, de tal forma que ya tenga un contenido de dulce considerable sin ser demasiado maduros pues esto acorta su vida en poscosecha, si se deja madurar mucho en árbol el proceso de clasificación, empaque y transporte podría dañar la fruta por acciones mecánicas, principal problema en posproducción (Campbell y Koch, 1989; Oslund y Davenport, 1983); este punto de madurez solo es recomendado para consumo interno en las fincas. Narain et al. (2001), Mitcham y McDonald (1991) afirman que el cambio de color de la corteza está ligado al aumento de la concentración de azúcares en carambolo siendo por tanto un buen indicador de madurez.

En Colombia los frutos pueden encontrarse en el árbol en diversas épocas del año aunque hay dos periodos anuales marcados en los que se encuentran las principales producciones estas son: de enero a marzo y de agosto a octubre. Similar comportamiento se encontró en Nayarit-México (Perez, Vasquez y Osuna, 2005), donde el primer periodo cosecha es entre febrero y marzo (correspondiente a la floración ocurrida en noviembre) y el segundo en los meses de septiembre y noviembre (floración de junio y julio). En condiciones de Florida, la recolección se hace generalmente en dos picos agosto a octubre, floración abril a mayo; y diciembre a febrero, correspondiente a floración septiembre-octubre (Al-Yahyai et al., 2005; Warren y Sargent, 2011; Crane, 1994). En Malasia se presentan picos de producción en abril-mayo, julio-agosto y noviembre-diciembre; en India el carambolo está disponible en septiembre-diciembre y diciembre-enero (Tongchitpakdee, 2012).

Para el mercado y embalaje de la carambola ácida del piedemonte amazónico se recomienda, según la tabla 1 presentada por González (2000), la recolección entre los índices 2 y 3 , cercano a los 80 y 94 días.

Hernández et al. (2004) afirman que el fruto debe ser manipulado cuidadosamente debido a su susceptibilidad a daños por golpes, manifestándose en la presencia de manchas superficiales de color café o agrietamiento de la corteza. Además el exceso de humedad estimula la aparición de hongos lo cual es evitado en el mercadeo, y frutos expuestos a periodos largos de refrigeración tienden a disminuir su aroma característico aunque limita el desarrollo de patógenos y la deshidratación (Hernández et al., 2004; Holman, 1998).

\section{CARAMBOLO EN EL PIEDEMONTE DEL META}

Para reconocer el comportamiento vegetativo del carambolo en condiciones de trópico hú-

Tabla 1. Carta de colores propuesta para el estado tres de desarrollo del fruto de carambola ácida del piedemonte amazónico.

\begin{tabular}{|c|l|l|}
\multicolumn{1}{|c|}{ Índice } & \multicolumn{1}{|c|}{ Color $^{1}$} & \multicolumn{1}{c|}{ Descripción } \\
\hline 1 & Amarillo-verde 1 & Color verde claro algo amarillo \\
\hline 2 & Amarillo-verde 2 & Color amarillo verdoso \\
\hline 3 & Pardo-naranja 1 & Color amarillo opaco \\
\hline 4 & Pardo-naranja 2 & Color naranja opaco poco intenso \\
\hline 5 & Pardo-naranja 3 & Color naranja opaco intenso, fruto completamente coloreado. \\
\hline
\end{tabular}

${ }^{1}$ Para la definición del color se tomó como referencia la RHS Colour Chart, donde los colores más aproximados fueron yellow-green group 146 y 163 y greyed-orange group 163 (tomado de González, 2000). 
medo se visitaron algunas fincas en tres zonas representativas de los suelos y condiciones climáticas del piedemonte del Meta (terrazas altas del piedemonte-Villavicencio, vegas del AriariGranada y Lejanías, y finalmente Restrepo), donde se tomaron las medidas de: altura de planta, copa y diámetro para determinar el volumen de copa. Aunque no todas las plantas tenían la misma edad, los valores se promediaron para obtener una imagen del comportamiento general de esta especie en condiciones locales.

Durante este ejercicio se pudo comprobar la buena adaptabilidad del carambolo en la región, encontrándose establecido en diversos paisajes sin ninguna dificultad (tabla 2).

De acuerdo con los resultados de la tabla 2 este frutal muestra un buen crecimiento y desarrollo en el piedemonte del Meta. Materiales evaluados en la vega del rio Ariari, presentan especial crecimiento comparado con los otros paisajes debido a la alta fertilidad de estos suelos, encontrando plantas con volúmenes de copa de hasta $110 \mathrm{~m}^{3}$ (figura 3) y $8 \mathrm{~m}$ de altura; plantas establecidas en suelos provenientes sabana presentan un desarrollo normal sin deficiencias aparentes. Se observó un menor perímetro del tallo en algunas de las plantas establecidas bajo sombra debido al elongamiento del tallo en busca de la radiación.
Se encontró además, según diálogo con los productores locales que plantas con 4 años de edad habían iniciado su ciclo productivo y plantas de 5-6 años de edad generaban producciones estables y constantes, con picos altos en los meses de enero, febrero, marzo, agosto y septiembre. Durante el trabajo de campo se encontraron además plantas de 35 años de edad, con aproximadamente 30 años de producción.

En la tabla 3 se encuentran los resultados de calidad de fruto de las muestras cosechadas en los 4 municipios del Meta, 5 frutos por árbol. De acuerdo con esto, en Restrepo los frutos tuvieron un mayor peso que en las demás localidades aunque en tamaño están muy parejos con Granada y Lejanías, mientras que es notable que en el Centro de Investigación La Libertad los frutos son de menor tamaño y peso. Las variaciones en calidad además puede deberse a que la etapa de madurez del fruto no era igual en las 5 localidades evaluadas.

En general los frutos de la región presentan un peso menor a los reportados por diversos autores. Perez et al. (2005) reportan para México frutos con pesos entre 125 y $147 \mathrm{~g}$; y longitud entre 11 y $12 \mathrm{~cm}$, el valor de diametro transversal es similar, y los SST estan enre los 8,5 y 9,6. Valores similares a los de Perez et al. (2005) son reportados por Goenaga (2007) para Puerto Rico en relacion a las variables de algura, diametro y SST.

Tabla 2. Comportamiento vegetativo de árboles de carambolo en cuatro municipios del departamento del Meta, Colombia.

\begin{tabular}{|c|c|c|c|l|} 
No. & $\begin{array}{c}\text { Altura planta } \\
(\mathrm{m})\end{array}$ & $\begin{array}{r}\text { Volumen de copa } \\
\left(\mathrm{cm}^{3}\right)\end{array}$ & $\begin{array}{r}\text { Edad promedio } \\
(\text { años })\end{array}$ & \multicolumn{1}{c|}{ Localidades } \\
\hline 4 & 4,40 & 7,11 & 10,0 & C.I. La Libertad, Villavicencio \\
\hline 2 & 5,50 & 27,14 & 11,0 & La Cosmopolitana, Restrepo \\
\hline 8 & 6,20 & 58,04 & 16,8 & Fincas, Granada \\
\hline 3 & 5,63 & 17,32 & 5,0 & Finca Los Naranjales, Lejanías \\
\hline 6 & 5,88 & 39,38 & 20,5 & Fincas, Lejanías \\
\hline
\end{tabular}




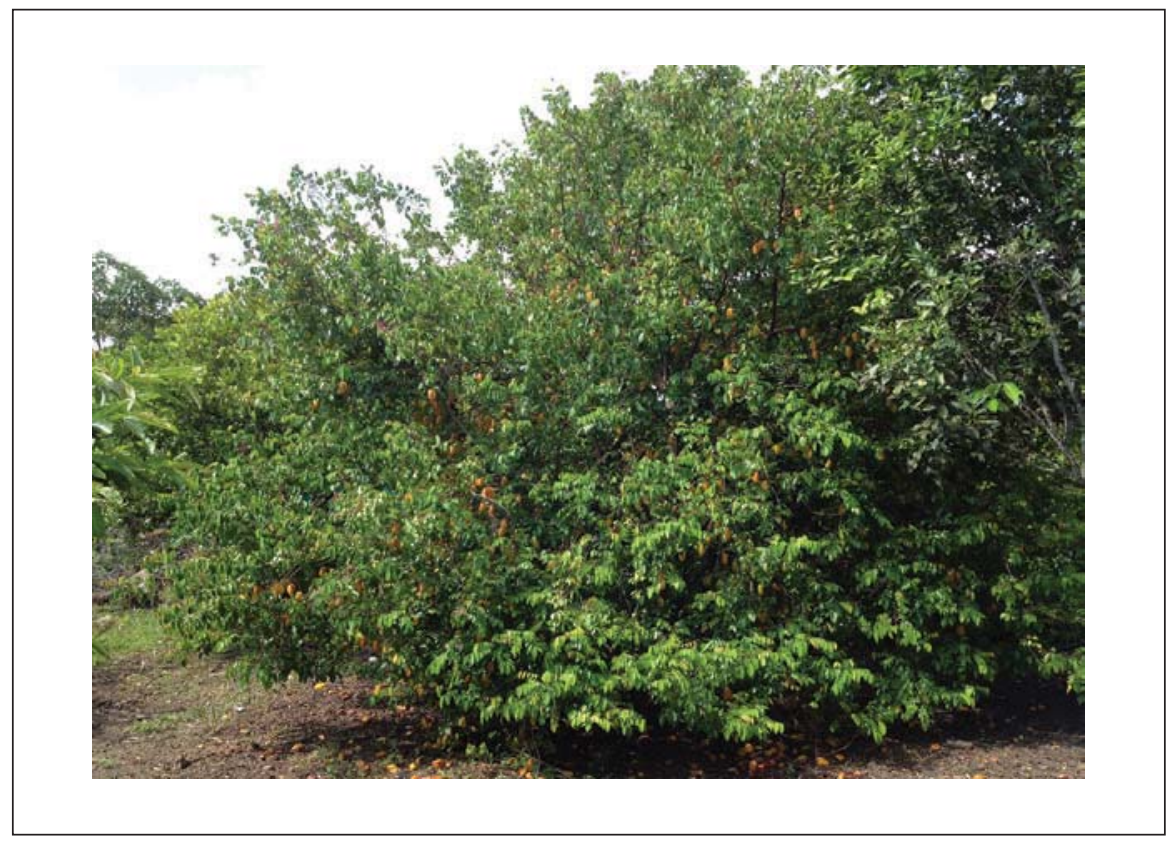

Figura 3. Plantas de carambolo en el municipio de Lejanías con alto desarrollo vegetativo. Foto: M.E. Arias C.

Tabla 3. Características físicas y químicas de frutos de carambolo en la región del piedemonte del Meta.

\begin{tabular}{|c|c|c|c|c|c|c|c|c|c|c|c|c|}
\hline $\begin{array}{l}\text { Peso de } \\
\text { fruto }(\mathrm{g})\end{array}$ & $\begin{array}{l}\text { Long. } \\
(\mathrm{cm})\end{array}$ & $\begin{array}{l}\emptyset \text { Trans. } \\
(\mathrm{cm})\end{array}$ & $\begin{array}{l}\text { Índice } \\
\text { de color }\end{array}$ & $\begin{array}{l}\text { Vol. de } \\
\text { jugo (ml) }\end{array}$ & $\begin{array}{l}\text { Peso de } \\
\text { jugo (g) }\end{array}$ & $\begin{array}{l}\text { Peso de } \\
\text { pulpa (g) }\end{array}$ & PH & $\begin{array}{l}\% \text { de } \\
\text { jugo }\end{array}$ & ATT & SST & $\mathrm{IM}$ & Localidades \\
\hline 72,36 & 7,99 & 5,69 & 2,25 & 49,70 & 84,72 & 17,30 & 2,86 & 67,90 & 0,45 & 7,39 & 16,51 & $\begin{array}{l}\text { C.I. La Libertad, } \\
\text { Villavicencio (árboles } 1 \text { a 4) }\end{array}$ \\
\hline 101,28 & 8,68 & 6,73 & 1,50 & 76,50 & 75,19 & 20,57 & 3,22 & 73,59 & 0,42 & 7,83 & 25,80 & $\begin{array}{l}\text { La Cosmopolitana, } \\
\text { Restrepo (5 y } 6)\end{array}$ \\
\hline 81,93 & 8,75 & 5,54 & 2,63 & 63,43 & 62,21 & 14,28 & 2,94 & 75,85 & 0,58 & 7,02 & 12,63 & $\begin{array}{l}\text { Fincas, Granada. } \\
\text { (7 a 14) }\end{array}$ \\
\hline 90,16 & 9,15 & 5,31 & 4,00 & 76,33 & 76,84 & 8,86 & 2,61 & 84,53 & 0,48 & 5,46 & 11,38 & $\begin{array}{l}\text { Los Naranjales, } \\
\text { Lejanías (15 a 17) }\end{array}$ \\
\hline 90,85 & 9,31 & 5,92 & 1,83 & 69,07 & 75,71 & 14,73 & 3,90 & 75,19 & 0,33 & 8,03 & 26,38 & $\begin{array}{l}\text { Fincas, Lejanías. } \\
\text { (18 a 23) }\end{array}$ \\
\hline
\end{tabular}

\section{Plagas y enfermedades de importancia en la región}

La principal plaga del carambolo es la mosca de las frutas (Anastrepha sp.), estas pueden ser controladas por medio de la utilización de trampas lumi- nosas, cebos, productos químicos o tratamientos de irradiación y frío (Paull y Chen, 2002). Agricultores de la región mencionaron también: la hormiga arriera (Atta sp.), los ácaros (Tetranychus sp.), entre otros organismos como, nematodos del género Rotylenchulus, escarabajos, etc. 
Cercospora averrhoae Petch. puede afectar seriamente las hojas y frutos con manchas, principalmente a aquellos árboles sometidos a estrés o deficiencia nutricional. Mientras que Botrytis spp., Alternaria spp., Colletotrichum spp., entre otros hongos pueden estar afectando la calidad externa de los frutos y a las flores (George, 1988; Watson et al., 1998)

Los productores de las fincas visitadas reportaron daños por pequeños cucarrones en frutos del municipio de Granada, Meta, en plantas bajo sombra y pendiente del $20 \%$, con incidencia de hongos y bacterias (figura 4A). Además se observó la presencia de fumagina en algunas de las plantas y frutos evaluadas sin ninguna clase de daños internos (figura 4B); algunos propietarios también reportan daños importantes por el ataque de hormiga arriera en la localidad de La Cosmopolitana, Restrepo, Meta.

En Colombia se estimó que la variedad Icambola producía cerca de 351,4 kg/año-árbol (Espinosa y González, 2014), promedio mucho mayor al de otros cultivares del país (Sánchez et al., 1985). En la actualidad se encuentra un único cultivo de importancia económica en la región del piedemonte del Meta ubicado en el municipio de Lejanías, vereda "Los Laureles" (trocha 22), finca "Los Naranjales", plantación de 5 años de edad, con un total de 70 plantas en producción, con rendimiento de hasta $290 \mathrm{~kg} /$ planta-año según Muñoz, administrador de la finca (comunicación personal); no se sabe con certeza si este material corresponde a la introducción ya mencionada Icambola. En Nayarit, México (trópico seco), el cultivar Fwang Tung alcanzó producciones medias de 150 kg/árbol, y el Maha 130 kg/árbol; en Florida se reportan producciones de 112-160 kg/árbol entre el año 7 y 12 (Pérez et al., 2004; Núñez y Crane, 1998).

\section{Comercialización y oportunidades de mercadeo}

Los frutos de carambolo pueden tener diversas posibilidades de mercadeo para los pequeños productores que son los que principalmente lo

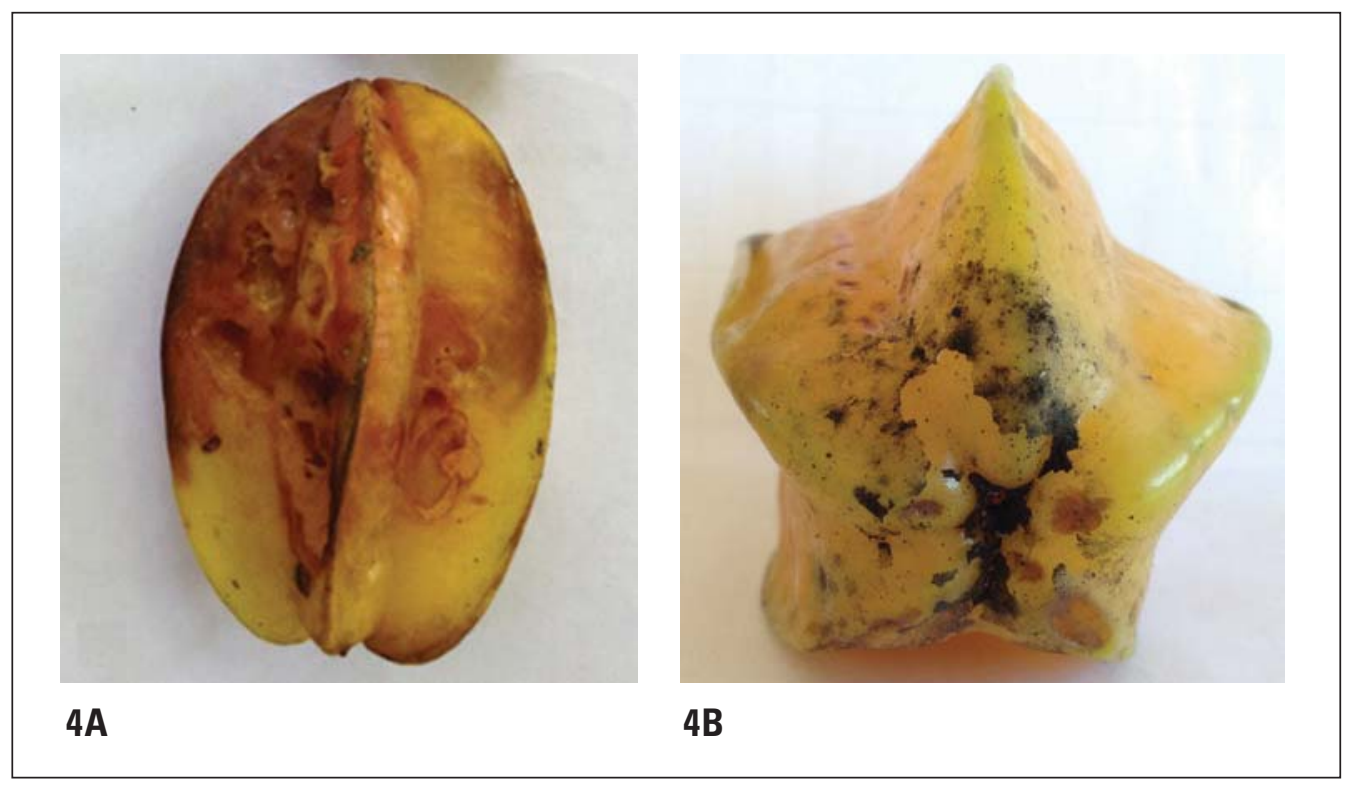

Figura 4. Daños más comunes en fruto de carambolo en condiciones del piedemonte del Meta (trópico bajo) A. Daño ocasionado por cucarrones, B. Capnodium. Fotos: H. Velásquez (4A) y M.E. Arias C. (4B). 
establecen en condiciones locales; en el Meta, el carambolo es consumido generalmente en fresco aunque también se emplean para preparación de jugo. En otros países estos frutos son vendidos como slices, cortes en rodajas empacados al vacío para ensaladas, y aderezos para bebidas o cocteles gracias a su forma llamativa, practicidad y beneficios a la salud (Patil et al., 2010). El principal atractivo nutricional de este producto es su actividad antioxidante y su contenido de ácidos grasos insaturados; los antioxidantes son las sustancias capaces de inhibir los procesos de oxidación para proteger al cuerpo del estrés oxidativo que puede desencadenar diversas enfermedades (Wei et al., 2014). Se ha encontrado también que el carambolo es rico en fibra dietaria, especialmente insoluble y que estas altas fracciones pueden ayudar a controlar la glucosa presente en la sangre después de las comidas (Patil et al., 2012), además de tener un contenido de humedad elevado, alrededor del 90,6\%, y minerales como calcio, potasio, fósforo, hierro (Márquez, et al., 2012).

En este aspecto, Teixeira et al. (2012) reportan que el principal limitante para la comercialización del carambolo en cortes frescos empacados al vacío es el rápido ablandamiento y oscurecimiento de la fruta, y que este daño está influenciado principalmente por la temperatura de almacenamiento. Encontraron que las temperaturas que favorecían la conservación de las rodajas eran las más bajas $\left(2,5\right.$ y $\left.5^{\circ} \mathrm{C}\right)$ mientras que temperaturas altas $\left(10^{\circ} \mathrm{C}\right)$ promovían el deterioro de los cortes con una correlación positiva con el ablandamiento y el metabolismo anaeróbico (concentración de $\mathrm{CO}_{2}$ ); este aspecto también es mencionado por Patil et al. (2010). Este tipo de avances en poscosecha deben ser tenidos en cuenta como oportunidad de innovación para ampliar el mercado de los productos provenientes de la agricultura familiar.

En Asia por ejemplo, esta fruta es consumida verde como un vegetal en ensaladas. Zainudin et al. (2014) determinaron que conocer el índice de madurez era esencial para obtener los máximos beneficios de la fruta; evaluando 5 épocas de cosecha (semana 9 a 13) encontraron que la semana 9 (frutos aún verdes) era aquella en la que se encontraban los mayores valores nutricionales en el fruto. Así mismo que el contenido total fenólico, el contenido total de flavonoides y beta-caroteno eran elevados en la fruta verde, mientras que el azúcar, el contenido total de carotenoides y beta-tocoferol predominaron en frutos maduros, en otras palabras se detectó que la actividad antioxidante decrecía a medida que el fruto adquiría madurez. De tal forma que el consumo de este fruto podría impulsarse en condiciones regionales por medio de presentaciones diferentes a la convencional como en Asia para ensaladas, aprovechando la ventaja que brinda la información científica respecto a los contenidos nutricionales de los frutos.

Zainudin et al. (2013) encontraron además que existían diferencias en los compuestos bioactivos y la actividad antioxidante entre cultivares de carambolo, evaluaron los B2, B10 y B17, cultivares comerciales más importantes de Malasia encontrando que los dos últimos exhibían mayor actividad antioxidante con diferencias significativas frente a B2, lo que sugiere la necesidad de modernizar este tipo de cultivos por medio de selección de material apropiado de acuerdo con el uso que tendrá y las prácticas de manejo adecuadas.

Actualmente este fruto se encuentra en fase de divulgación y promoción en diferentes países del mundo. Warren y Sargent (2011) aseguran que el carambolo se encuentra entre los cuatro frutales tropicales más conocidos o familiares para los consumidores de Florida. Europa es un mercado potencial, así como algunos países asiáticos y latinos donde el fruto ha venido ganando lugar en mercados mayoristas, no solo en fresco sino también en pulpa (Escobedo, 2010).

En Cauca, Colombia, uno de los principales departamentos productores de carambolo, a la pla- 
za de mercado el 70\% del producto llega en caja de cartón corrugado y el 30\% en canastilla plástica (Sánchez et al., 2011). Mientras que se manejan principalmente dos empaques de venta: bandejas de icopor recubiertas con una película plástica transparente en cantidades de $1 \mathrm{lb}$ o de $1 \mathrm{~kg}$, este tipo de empaque corresponde al 70\%, mientras que el $30 \%$ se hace en bolsa plástica de acuerdo con la cantidad del producto pedido por el cliente. En Lejanías, Meta, el fruto es recolectado y empacado en canastillas plásticas de 25 kg y vendido en la central de abastos de Bogotá con un precio de 12 mil pesos canastilla, con un mercado de gran aceptación por la calidad de la fruta.

\section{CONCLUSIÓN}

El carambolo es un frutal introducido de Asia que ha presentado una sobresaliente adaptación en las condiciones del trópico bajo de Colombia, en donde se cultiva en la mayoría de los departamentos que tienen estas condiciones climáticas en el país. El mejoramiento del mercadeo de la fruta con selección de clones de mayor demanda, la adecuación de empaques y el desarrollo de presentaciones y formas de preparaciones novedosas permitirán ampliar el consumo para beneficio de los productores que en su totalidad pertenecen a la agricultura familiar.

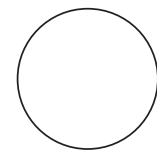

Al-Yahyai, R. 2004. Soil water depletion, growth, physiology, and yield of carambola trees in krome soil. University of Florida, Gainesville, FL.

Al-Yahyai, R., B. Schaffer, F. Davies y J.H Crane. 2005. Four levels of soil water depletion minimally affect carambola phonological cycles. HortTechnol. 15(3), 623-630.

Bouzayen, M., A. Latché, P. Nath y J.C. Pech. 2010. Mechanism of fruit ripening. pp. 319-339. En: Pua E.C. y M.R. Davery (eds.). Plant developmental biology - Biotechnological perspectives. Vol. 1. Springer, Berlín.

Campbell, C.A. y K.E. Koch. 1989. Sugar/acid composition and development sweet and tart carambola fruit. J. Amer. Soc. Hort. Sci. 114, 455-457.

Campbell, C.W., R.J. Knight y R. Olszack. 1985. Carambola production in Florida. Proc. Fla. State Hort. Soc. $98,145-149$.

Campbell, C.W. y S.E. Malo. 1981. The carambola. Fla. Coop. Ext. Serv. Fruit Crops Fact Sheet FC-12. Gainesville, FL.

Campbell, C.W. y R.J. Marte. 1990. Pre-production, production and post-harvest handling of carambola. Miscellaneous Publication IICA, Bridgetown, Barbados.

\section{REFERENCIAS BIBLIOGRÁFICAS}

Crane, J.H. 1994. The carambola (star fruit). Fact Sheet HS-12. Florida Cooperative Extension Service, IFAS, University of Florida, Gainesville, FL.

Enciclopedia Agropecuaria Terranova. 1995. Producción Agrícola I. Terranova Editores, Bogotá.

Escobedo, M. 2010. Sondeo rápido de mercado; pulpa de frutas. CATIE, Turrialba, Costa Rica.

Espinosa, A.M. y Y. González. 2014. Efecto de la deshidratación por aire caliente sobre las características morfométricas y colorimétricas de estrellas de carambolo (Averrhoa carambola). Trabajo de grado. Facultad de Ingeniería, Fundación Universitaria Agraria de Colombia, Bogotá.

Galán, S. y U.G. Menini 1993. Carambola cultivation. FAO Plant Production and Protection Paper No. 108. FAO, Roma.

George, A.P. y R.J. Nissen. 1994. Carambola. pp. 206211. En: Schaffer, B. y P. Andersen (eds.). Handbook of environmental physiology of fruit crops. Vol. 2: Sub-tropical and tropical Crops. CRC Press, Boca Raton, FL.

George, A.P., R.J. Nissen y B.J. Watson. 1988. Varietal evaluation and selection in carambola. Proc. $4^{\text {th }}$ Aust. Conf. on fruit and nuts. Exotic Fruit Growers Association, Lismore, Australia. 
Goenaga, R. 2007. Yield and fruit quality of carambola cultivars grown at three locations in Puerto Rico. HortTechnol. 17(4), 604-607.

González, D.V. 2000. Análisis del desarrollo de la fase reproductiva y determinación de parámetros de recolección de la carambola (Averrhoa carambola L.) variedad ácida, producida en el piedemonte amazónico colombiano. Trabajo de grado. Facultad de Agronomía, Universidad Nacional de Colombia, Bogotá.

González, V., M. Hernández, A. Herrera, J. Barrera, O Martínez y D. Páez. 2001. Desarrollo del fruto e índices de cosecha la carambola (Averrhoa carambola L.) producida en el piedemonte amazónico colombiano. Agron. Colomb. 18(1-2), 7-13.

Green, J.G. 1987. Carambola production in Malaysia and Taiwan. Proc. Fla. State Hort. Soc. 100, 275 278.

Hernández, M.S., J.A. Barrera, D.O. Páez, E. Oviedo y H. Romero. 2004. Aspectos biológicos y conservación de frutas promisorias de la Amazonía colombiana. Instituto Amazónico de Investigaciones Científicas (SINCHI), Bogotá.

Hii, C.L. y J.F. Ogugo. 2014. Effect of pre-treatment on the drying kinetics and product quality of star fruit slices. J. Eng. Sci. Technol. 9(1), 122-134.

Holman, R. 1998. StarFruit. Sheridan Fruit Company, Inc. Portland, OR.

Illescas, J.L., O. Bacho y S. Ferrer. 2007. Análisis de los principales frutos tropicales comercializados. Mercasa. En: http://www.mercasa.es/files/multimedios/1288280121_DYC_2007_95_33_85.pdf; consulta: junio de $201 \overline{4}$.

Janick, J y R. Paull. 2008. The encyclopedia of fruit and nuts. CABI Publishing, Wallingford, UK.

Knight, R.J. 1965. Heterostyly and pollination in carambola. Proc. Fla. State Hort. Soc. 78, 375-378.

León, J. 2000. Botánica de los cultivos tropicales. $3^{\text {a }}$ ed. Editorial Agroamerica, IICA, San José.

Litz, R.E. y J.L. Griffis. 1989. Carambola (Averrhoa carambola). Biotechnol. Agr. For. 5, 59-67.

Loor, M.R. 2013. Aplicación de tecnología de procesamiento mínimo en la conservación de fruta china (Averhoa carambola L.) empacada al vacío. Trabajo de grado. Universidad Laica Eloy Alfaro de Manabi, Manta, Ecuador.

MACIA (Ministerio de Asuntos Campesinos, Indígenas y Agropecuarios). 2003. Estudio cadena productiva: Frutas exóticas. Cochabamba, Bolivia.
Márquez, L., C. Pretell y C. Minchón. 2012. Efecto de la dosis de irradiación UV-C y tiempo de almacenamiento sobre las características fisicoquímicas, microbiológicas, y antioxidantes en rebanadas de carambola (Averrhoa carambola L.), variedad Golden Star mínimamente procesada. Pueblo Cont. 23(2), 353-369.

Mendoza, J.A. y F.L. Ramírez. 1997. Pequeños productores, grandes negocios. El potencial económico de los productores agropecuarios comercialmente no tradicionales. Memoria de la Primera Exposición Nacional, México.

Mitcham, E.J. y E.E. McDonald. 1991. Characterization of the ripening of carambola (Averrhoa carambola L.) fruit. Proc. Fla. State Hort. Soc. 104, 104-108.

Morton, J. 1987. Carambola. En: Fruits of warm climates. Southern Book Service. Miami, FL.

Nakasone, H.Y. y R.E. Paull, 1998. Tropical fruits. CAB International, Wallingford, UK.

Narain, N., P.S. Bora, H.J. Holschuh y M.A. Vasconcelos. 2001. Physical and chemical composition of carambola fruit (Averrhoa carambola L.) at three stages of maturity. Cienc. Tecnol. Aliment. 3(3), 144-148.

Navarro, B. 2011. Análisis bromatológico del carambolo (Averrhoa carambola L.) y determinación de su capacidad antioxidante. Tesis. Universidad Veracruzana. México D.F.

Ngah, W.A., I. Ahmad y A. Hassan, 1989. Carambola production, processing and marketing in Malaysia. Proc. Interam. Soc. Trop. Hort. 33, 30-43.

NIIR Board. 2005. Cultivation of fruits, vegetables and floriculture. National Institute of Industrial Research Board, New Delhi.

Novillo, G.C. 2009. Desarrollo y evaluación física, química y sensorial de jugo de dos variedades de carambola (Averrhoa carambola). Trabajo de grado. Escuela Agrícola Panamericana, Zamorano, Honduras.

Núñez, R. y J.H. Crane. 1998. Phenology, shoot development, and floral initiation of carambola (Averrhoa carambola L. cv. Arkin) in a subtropical environment. Proc. Fla. State Hort. Soc. 111, 310-312.

Núñez-Elisea, R. y J.H. Crane, 2000. Selective pruning and crop removal increase early-season fruit production of carambola (Averrhoa carambola L.). Sci. Hortic. 86, 115-126.

O’Hare, T.J. 1997. Carambola. pp. 295-307. En: Mitra, S.K. (ed.). Postharvest physiology and storage of tropical and subtropical fruits. Cabi Publishing, Oxford, UK. 
O'Hare, T.J. 1993. Postharvest physiology and storage of carambola (starfruit): a review. Postharvest Biol. Technol. 2(4), 257-267.

Orduz, J.O. y J.A. Rangel. 2002. Frutales tropicales potenciales para el piedemonte llanero. Manual de asistencia técnica No. 8. Corpoica y Produmedios, Villavicencio, Colombia.

Orwa, C., A. Mutua, R. Kindt, R. Jamnadass y S. Anthony. 2009 Agroforestree Database:a tree reference and selection guide version 4.0. World Agroforestry Centre. En: http://www.worldagroforestry.org/treedb/AFTPDFS/Averrhoa_carambola. pdf; septiembre de 2014.

Oslund, C.R y T.L Davenport. 1983. Ethylene and carbon dioxide in fruit ripening of Averhoa carambola. HortScience 18, 229-230.

Pérez, M.H., V. Vázquez y J.A. Osuna. 2005. El cultivo del carambolo (Averrhoa carambola L.): una alternativa para el trópico seco. Rev. Chapingo Ser. Hort. 11(1), 83-87.

Patil, A.G, S. Koli, D. Patil y A. Phatak. 2012. A comprehensive review of an important medicinal plant - Averrhoa carambola L. Pharmacognosy Comm. 2(2), 13-17.

Patil, A.G., D.A. Patil, A.V. Phatak y N. Chandra. 2010. Physical and chemical characteristics of carambola (Averrhoa carambola L.) fruits at three stages of maturity. IJABPT 1(2), 624-629.

Paull, R.E. y C.C. Chen. 2002. Carambola. En: The commercial storage of fruits, vegetables, and florist and nursery crops. Agricultural Handbook 66. US Department of Agriculture, Washington DC.

Paull, R.E. y O. Duarte. 2012. Tropical fruits. Vol. 2. CAB International, Wallingford, UK.

Ray, P.K. 2002. Breeding tropical and subtropical fruits. Narosa Publishing House, New Delhi.

Ruehle, G.D. 1958. Miscellaneous tropical and subtropical Florida fruits. Fla. Agr. Ext. Serv. Bul. 156A, 30-33.

Saghir, S., A. Sadikum, K.Y. Khaw y V. Murugaiyah, 2013. Star fruit (Averrhoa carambola L.): From traditional uses to pharmacological activities. Bol. Latinoam. Caribe Plant Med. Aromat. 12(3), 209-219.

Sánchez, E.F., L.A Rivera y J.M. Tobar. 2011. Comercialización de productos vegetales en la plaza de mercado del barrio bolívar de la ciudad de Popayán-Cauca (Colombia). En: http://vegetalespopayan.jimdo.com/ prodvegetales/carambolo/; consulta: mayo de 2011.
Sánchez, L.A., R. Torres, R. Salazar y D. Ríos-Castaño. 1985. Comportamiento de cuarenta frutales tropicales no explotados comercialmente en Colombia. Rev. ICA 20, 1-12.

Sharma, G., O.C. Sharma y B.S. Thakur. 2009. Systematics of fruit crops. New India Publishing Agency, New Delhi.

Teixeira, G., J.F. Durigan, A.S. Ferraudo, R.E. Alves y T.J. O'Hare, 2012. Multivariate analysis of freshcut carambola slices stored under different temperatures. Postharvest Biol. Technol. 63, 91-97. Doi: 10.1016/j.postharvbio.2011.09.005

Tongchitpakdee, S. 2012. Longan and carambola. pp. 223-240. En: Tropical and subtropical fruits. Postharvest physiology, processing and packaging. Wiley-Blackwell, New York, NY.

Villegas, B.E. 1998. El carambolo (Averrhoa carambola). En: Seminario Regional de Especies Vegetales Promisorias. Facultad de Ciencias Agropecuarias, Universidad Nacional de Colombia, Medellín, Colombia.

Wagner, C.J., W.L. Bryan, R.E. Berry y R.J. Knight. 1975. Carambola selection for commercial production. Proc. Fla. State Hort. Soc. 88, 466-469.

Wahab, A., I.A. Ahmad y A. Hassan. 1989. Carambola production, processing and marketing in Maysia. Proc. Interam. Soc. Trop. Hort. 33, 30-43.

Warren, O. y S.A. Sargent. 2011. Carambola (Averrhoa carambola L.). pp. 397-413. En: Yahia, E.M. (ed.). Postharvest biology and techology of tropical and subtropical Fruits. Vol. 2. Woodhead Publishing, Oxford, UK.

Watson, B.J., A.P. George, R.J. Nissen y B.I. Brown. 1998. Carambola: a star on the horizon. Queensl Agr. J. 114, 45-51.

Wei, S.D., H. Chen, T. Yan, Y.M. Lin y H.C. Zhou. 2014 Identification of antioxidant components and fatty acid profiles of the leaves and fruits from Averrhoa carambola. LWT - Food Sci. Technol. 55(1) 278-285. Doi:10.1016/j.lwt.2013.08.013

Zainudin, M.A., A.A. Hamid, F. Anwar, A. Osman y N. Saari. 2013. Variation of bioactive compounds and antioxidant activity among three cultivars (B2 $\mathrm{B} 10$ and B17) of carambola (Averrhoa carambola L.) fruits. Agrochimica 57(3), 264-278.

Zainudin, M.A., A.A. Hamid, F. Anwar, A. Osman y N. Saari, 2014. Variation of bioactive compounds and antioxidant activity of carambola (Averrhoa carambola L.) fruit at different ripening stages. Sci. Hortic. 172(9), 325-331. Doi: 10.1016/ j.scienta.2014.04.007 